\title{
Dietary fish oil and the severity of symptoms in patients with systemic lupus erythematosus
}

\author{
A J E Walton, M L Snaith, M Locniskar, A G Cumberland, W J W Morrow, D A Isenberg
}

\begin{abstract}
A prospective, double blind, cross over study assessing the effects of a low fat, high marine oil diet in 27 patients with active systemic lupus erythematosus has been performed. The patients were given $20 \mathrm{~g}$ daily of MaxEPA (eicosapentaenoic acid) or $20 \mathrm{~g}$ of olive oil (placebo) in matching capsules added to a standardised isoenergetic low fat diet. When individual outcome measures of the 17 patients who completed the full 34 week study were considered 14 who were receiving MaxEPA achieved useful or ideal status, whereas 13 receiving placebo were rated as worse or no change. The difference between the two types of capsule was statistically significant. No major side effects were noted, and it is suggested that dietary modification with additional marine oil may be a useful way of modifying disease activity in systemic lupus erythematosus.
\end{abstract}

In the past 10 years several studies on mouse models have indicated that there may be a role for dietary modification in the management of autoimmune diseases. ${ }^{1}$ In particular, diets containing high concentrations of fish oil may be especially beneficial in suppressing disease. For example, the effects of a diet rich in eicosapentaenoic acid (an omega-1 class fatty acid typically found in fish oil) upon nephritis in female $(\mathrm{NZB} \times \mathbf{N Z W}) \mathrm{F}_{1}$ mice which spontaneously develop an autoimmune syndrome similar to human systemic lupus erythematosus have been described. ${ }^{2}$ In that study only $15 \%$ of the mice given the diet from weaning died from renal disease at 19 months of age as compared with $98 \%$ of a control group fed on a beef tallow diet. Increased longevity was also achieved when the diet was instituted at 4-5 months of age. Similarly, a reduction in the progression of established renal disease in NZB/W mice, and a delay of renal disease in BXSB and MRL-mp-lpr-/lpr (MRL/lpr) strains were shown when mice were fed a diet containing marine lipid ( $25 \%$ menhaden oil). ${ }^{3}$

Supplementation of the diet of MRL/lpr mice with fish oil as the exclusive source of lipid was found to suppress features of lupus, possibly owing to a change in endogenous cyclooxygenase metabolite synthesis. ${ }^{4}$ Godfrey and colleagues $^{5}$ studied the effects of dietary fatty acid on various disease parameters in the autoimmune MRL/lpr mouse model of systemic lupus erythematosus, using a variety of oils (olive, sunflower, evening primrose oil, fish, and evening primrose oil/fish mixture). Both the mice receiving the evening primrose oil and the evening primrose oil/fish oil mixture showed an increase in survival.

These studies indicate the potential use of dietary supplementation for the management of human autoimmune disease, ${ }^{6}$ but to date there have been only a few studies of rheumatoid arthritis and systemic lupus erythematosus. In one unblinded study the diets of 12 patients with active rheumatoid arthritis were supplemented with $20 \mathrm{~g}$ of MaxEPA (eicosapentaenoic acid) fish oil for six weeks, and the clinical results suggested that the supplement might have an anti-inflammatory effect. ${ }^{7}$

In a more recent placebo controlled blind study dietary manipulation of foods to which patients with rheumatoid arthritis might be intolerant showed that there was significant objective improvement during the periods of dietary therapy compared with periods of placebo treatment. ${ }^{8}$ It has also been reported that requirements for non-steroidal antiinflammatory drugs in patients with rheumatoid arthritis were reduced when dietary essential fatty acids were altered by the inclusion of evening primrose oil or fish oil containing eicosapentaenoic acid. ${ }^{9}$

We now report a double blind, cross over study of 27 patients with systemic lupus erythematosus using a low fat diet with the addition of MaxEPA or olive oil. The purpose of this study was to determine the effect of a low fat eicosapentaenoic acid enriched diet on the course of disease in patients with systemic lupus erythematosus.

\section{Patients, materials and methods PATIENTS}

Patients with established systemic lupus erythematosus attending the Bloomsbury rheumatology unit clinic were seen and offered the opportunity to take part in the trial, provided that they were not severely ill and thus unable to participate in the study or were clinically inactive; patients with major renal disease already receiving special diets were also not considered suitable. Each of the patients in the study met four or more of the revised criteria for the classification of systemic lupus erythematosus suggested by the American Rheumatism Association. ${ }^{10}$ When initial interest was expressed by any patient their eating habits were discussed with them and an outline of the proposed diet was explained with further advice tailored to suit the individual. The patients were then given the opportunity to study the diet before coming to any decision. 
Thirty seven patients were offered the chance to take part in the study, of whom 27 (25 female, two male) expressed interest and were found to be suitable upon further investigation. Their ages ranged from 21 to 68 years at the start of the study. The patients manifested the wide range of clinical features and serological abnormalities typical of systemic lupus erythematosus.

DIET

The diet forming the main base of the study was a low fat, isoenergetic diet designed to provide not more than $20 \%$ energy from fat a day. Fats from both animal and vegetable sources were excluded or restricted, and emphasis placed on increasing the carbohydrate-rich foods. Patients who were overweight were encouraged not to look upon the diet as a means to losing weight, though they could reduce their energy intake to not less than $6270 \mathrm{~kJ}$ a day.

\section{CAPSULES}

Because of the low fat diet patients were given combined vitamin A and D capsules BPC, as a precautionary measure: one daily was taken throughout the period of the study. Each capsule provided 4000 IU vitamin A and 400 IU vitamin D.

Fish oil was given for 12 weeks during the study, supplied in the form of opaque capsules (MaxEPA, Marfleet Refining, Hull), each containing $1 \mathrm{~g}$ oil flavoured with peppermint. Twenty capsules were taken each day in divided doses.

The control capsules (Marfleet Refining, Hull), which were given for a further 12 week period, were identical in appearance to the fish oil capsules and contained peppermint flavoured olive oil.

Capsules were randomly allocated in a double blind manner so that neither the patients nor the doctors (DAI, MLS) assessing the patients were aware of which capsules were being taken. Only the dietitian (AW) knew the allocation, but she did not participate in the assessment of disease activity. At each patient assessment full clinical and serological data were collected.

\section{TRIAL DESIGN}

On entry into the study a detailed dietary history of each patient was taken by recall. The patient's understanding of the proposed diet was then checked and the patient entered into the study. The proposed length of $\mathbf{3 4}$ weeks for the study was divided into sections (table 1). The initial two week period was used as a run-in period to check the patients understanding and

Table 1 Treatment pattern for the patients with systemic lupus erythematosus

\begin{tabular}{ll}
\hline Week & Treatment \\
\hline $0-$ & Diet only \\
$2-$ & Diet +capsule a/b \\
$14-$ & Diet only $\quad$ Diet + capsule b/a \\
$22-34$ & . \\
\hline
\end{tabular}

compliance with the diet. If necessary this period was extended to help the patient adjust to the diet more fully. The patient then started taking one of the sets of capsules.

\section{PATIENT ASSESSMENT}

At the initial appointment and then at fourweekly intervals thereafter each patient was seen for a full medical and dietetic assessment. The latter was carried out to ensure that the patients were adhering to their diet. Blood samples were taken at each appointment, both for routine tests (full blood count, $\mathrm{C} 3$, and antidouble stranded DNA antibody levels) and also for red cell fatty acid analysis.

At intervals throughout the study patients were asked to fill in a daily food chart, which was later calculated to give figures for total energy intake and percentage fat especially. This was used as a check on compliance and understanding of the diet.

A set of criteria was established for each patient on the basis of their individual clinical state when they started each set of capsules, indicating what would be considered ideal improvement', 'useful improvement', 'no change', and 'deterioration' after 12 weeks. Full details of this method have been published elsewhere. ${ }^{11}$ Ideal improvement was intended to indicate the best possible outcome which might be expected in the face of irreversible problems, such as chronic uraemia or joint deformity. Useful improvement was intended to indicate an improvement short of ideal without any new clinical features. A static state was intended to indicate the absence of either useful improvement or significant deterioration. Significant deterioration was intended to imply that existing clinical problems had exacerbated or that new problems had developed that were of greater importance than any coexisting improvement.

The criteria for outcome were different for each patient, based on the relevance to that individual. Criteria were often quite complex, being derived from a range of baseline clinical and laboratory data. Table 2 gives an example of a set of criteria. Full details of every subject's criteria are available from the authors.

Table 2 Criteria for patient 12

\section{First phase}

1 No active rash

2 No joint pain

3 No lethargy

4 No mouth ulcers

5 Erythrocyte sedimentation rate $<30 \mathrm{~mm} / \mathrm{h}$

6 Visual analogue score of patient's wellbeing $<2 \mathrm{~cm}$

7 No new features of systemic lupus erythematosus

Ideal $6 / 7$; useful $4 / 7$

Second phase

1 Visual analogue score of patient's wellbeing $<2 \mathrm{~cm}$

2 No rash

3 No deep venous thrombosis or neurological even

4 No shortness of breath

5 Total white blood cell count $>4.0 \times 10^{9} / \mathrm{l}$

6 Total lymphocyte count $>1.5 \times 10^{9} / 1$

7 Erythrocyte sedimentation rate $>45 \mathrm{~mm} / \mathrm{h}$

Ideal $6 / 7$; useful 3-4/7

Note: The clinical features referred to above are those to which this particular patient was prone. 
EXTRACTION OF RED BLOOD CELL LIPIDS AND GAS CHROMATOGRAPHIC ANALYSIS

Blood samples $(5 \mathrm{ml})$ were collected in EDTA. The plasma and buffy coat were removed and the red blood cells washed twice with saline. Washed packed red blood cells $(1 \mathrm{ml})$ were stored at $-70^{\circ} \mathrm{C}$ until analysed. The cells were then thawed and the lipids extracted by the method of Folch et al. ${ }^{12}$ Butylated hydroxytoluene was added to the methanol $(50 \mathrm{mg} / \mathrm{l})$ to prevent autoxidation of the lipids. Fatty acid methyl ester derivatives ${ }^{13}$ were analysed with a Varion (Sunnyvale, CA, USA) model 3400 gas chromatograph equipped with a flame ionisation detector and a 60 metre SP-2330 megabore column (Supelco, Bellafonte, PA, USA). The column temperature was programmed at $150^{\circ} \mathrm{C}$ for 10 minutes, increased by $3^{\circ} \mathrm{C} / \mathrm{min}$ to $190^{\circ} \mathrm{C}$, and held for 15 minutes. Fatty acid identification was made by comparison of retention times with authentic standard fatty acids mixtures (Sigma Chemical, St. Louis, MO, USA and Supelco) analysed under the same conditions.

\section{Results}

Of the 27 patients who started the study, 10 dropped out at various stages. Four patients dropped out before starting the capsules owing to an inability to cope with the low fat diet as part of their lifestyle. A further four patients started the capsules but dropped out of the study soon afterwards for reasons varying from inability to swallow the capsules to nonattendance at clinic appointments. One patient who had gallstones was vomiting back the cap-

Table 3 Results of treatment with MaxEPA (eicosapentaenoic acid) of 17 patients with systemic lupus erythematosus

\begin{tabular}{lll}
\hline MaxEPA $\rightarrow$ placebo & Receiving MaxEPA & Receiving placebo \\
& 0 & 0 \\
Ideal & 6 & 0 \\
Useful & 2 & 7 \\
Static & 0 & 1 \\
Worse & \\
\multicolumn{2}{l}{ Placebo $\rightarrow$ MaxEPA } & \\
Ideal & Receiving placebo & Receiving MaxEPA \\
Useful & 1 & 3 \\
Static & 3 & 5 \\
Worse & 5 & 1 \\
& 0 & 0 \\
\hline
\end{tabular}

When the ideal and useful categories were combined and compared with the static and worse groups combined the difference in outcome between MaxEPA and the control capsules was significant $\left(\mathrm{p}<0.01\right.$ by $\chi^{2}$ analysis with Yates's correction for small numbers)

Table 4 Maximum percentage change in eicosapentaenoic acid concentrations

\begin{tabular}{lcc}
\hline Patient code & $\begin{array}{l}\text { Receiving MaxEPA } \\
(\%)\end{array}$ & $\begin{array}{l}\text { Receiving olive oil } \\
(\%)\end{array}$ \\
\hline 2 & $400 \cdot 0$ & $28 \cdot 6$ \\
5 & $544 \cdot 4$ & 0 \\
9 & $139 \cdot 1$ & $4 \cdot 3$ \\
10 & $77 \cdot 8$ & $-55 \cdot 6$ \\
11 & $562 \cdot 5$ & $2 \cdot 5$ \\
12 & $51 \cdot 5$ & $-153 \cdot 8$ \\
13 & $285 \cdot 7$ & $42 \cdot 8$ \\
15 & $51 \cdot 4$ & Not done \\
16 & $104 \cdot 5$ & $18 \cdot 2$ \\
17 & $133 \cdot 3$ & $16 \cdot 6$ \\
18 & $671 \cdot 4$ & $71 \cdot 4$ \\
20 & $328 \cdot 6$ & $14 \cdot 3$ \\
21 & $168 \cdot 7$ & $18 \cdot 7$ \\
22 & $66 \cdot 6$ & Not done \\
23 & $10 \cdot 0$ & 0 \\
26 & $411 \cdot 1$ & $11 \cdot 1$ \\
\hline
\end{tabular}

sules, and therefore was withdrawn from the study. One patient completed the first phase of the study and was judged to have had a useful result, but was weak and unable to continue owing to a rapid deterioration in her condition. She was admitted as an inpatient with severe diarrhoea, which was later found to be due to campylobacter infection.

Table 3 shows the results obtained for the remaining patients. It is evident that those patients receiving the MaxEPA and the diet did significantly better than those receiving diet and control capsules $(p<0.01)$. For example, whereas 14 patients were recorded as having achieved useful/ideal status with MaxEPA plus diet, only four did so with control capsules plus diet. As a corollary, 13 patients receiving placebo plus diet were rated static/worse compared with three receiving MaxEPA plus diet.

Table 4 shows the maximum change in red cell eicosopentaenoic acid concentrations while patients were receiving MaxEPA and olive oil. In all, 16 patients had sufficient numbers of blood tests and suitable sample preparations for this estimation. With the exception of patient 23 , in whom little change in the concentrations was noted while receiving MaxEPA or olive oil, all the remaining patients in whom a comparison could be made showed a substantial increase in their red cell eicosapentaenoic acid concentration while receiving MaxEPA.

\section{Discussion}

This study clearly shows the potential for diet in the treatment of systemic lupus erythematosus. Although not every patient could adhere strictly to the diet or manage the capsules, the improvement in those who could do both was statistically significant.

We are unaware of any previous well controlled double blind study performed in this way in patients with systemic lupus erythematosus. The previous attempts to treat autoimmune rheumatic diseases with dietary modification seem mostly to have been restricted to patients with rheumatoid arthritis but have attracted considerable attention. ${ }^{14}$ Thus a diet popularised in the lay press, ${ }^{15} 16$ and which excludes dairy products, meat, spices, alcohol, additives, and preservatives, failed to show an objective overall clinical benefit when compared with a placebo diet in a group of patients with longstanding, progressive, active rheumatoid arthritis. ${ }^{17}$ However, a more recent study in dietary manipulation using foods least likely to cause intolerance (an elimination diet), with the reintroduction of further foods, showed a significant objective improvement during the periods of diet treatment, ${ }^{8}$ as indicated by an improvement in the numbers of patients with severe pain, numbers of painful joints, erythrocyte sedimentation rate, and platelet count.

Recent interest has centred on the influence of the manipulation of dietary fatty acids, particularly polyunsaturated fatty acids, with the addition to the diet of evening primrose oil or fish oil or a combination, ${ }^{18}$ with one study showing a decrease in the amount of nonsteroidal anti-inflammatory drugs used to treat 
rheumatoid arthritis." Studies in patients with rheumatoid arthritis whose diets were supplemented with eicosapentaenoic acid or fish oil showed some beneficial effects, with a reduction in the number of tender joints. ${ }^{19} 20$ A further study showed statistically significant improvement for joint swelling and duration of early morning stiffness, while other clinical variables (joint swelling index, visual analogue pain scale, and grip strength) favoured the fish oil over the placebo. ${ }^{21}$ Thorner and colleagues ${ }^{22}$ described the treatment of 19 patients with systemic lupus erythematosus in an open study by reducing the polyunsaturated fat in the diets (and increasing the saturated fat). The number of patients with active systemic lupus erythematosus was reduced from 11 to three by the end of the year long study and a modest reduction in prednisolone was achieved. The report gives no real indication of how disease activity was assessed, however, and a simple placebo effect cannot be ruled out.

There is no uniform method of assessing disease activity in lupus. As Liang and colleagues have discussed, over 60 different attempts have been described. ${ }^{23}$ Most are based on the premise that the more systems affected, the more active is the disease. This seems to us much too simplistic as, for example, a patient with a mild skin rash, modest arthralgia, and a pleural rub would be scored as more active than a patient with severe, life threatening disease restricted to her kidneys. The other common tendency is to use indices which make little or no allowance for the rate of changes in symptoms or signs and provide a single 'total' disease activity score. This may also be misleading as it says nothing about the degree to which different systems are affected. We and others are working towards the goal of accurate sensitive measurement of disease activity in systemic lupus erythematosus. ${ }^{24}$ In this study an individualised outcome method was used as described previously. ${ }^{11}$ We believe that given the highly variable nature of lupus this is more likely to provide a realistic assessment of the degree of improvement that might be expected in any given lupus patient. The method awaits formal validation, however.

Precisely how dietary manipulation might be beneficial remains uncertain. A recent review of the available evidence from the animal models studied suggests a number of possible mechanisms. ${ }^{25}$ For example, it has been shown that prostaglandins and leukotrienes synthesised from eicosapentaenoic acid have an immunoinhibitory effect. Furthermore, it has been shown that fish oil contains high quantities of omega-3 fatty acids, which are extensively incorporated into several classes of lipid in the spleens of autoimmune mice given a fish oil diet. ${ }^{26}$ This lipid substitution might result in altered production of platelet activating factor and synthesis of eicosapentanoids derived from arachidonic acid, thus exerting antiinflammatory effects.

Although this study is confined to small numbers, its implications are significant and a larger study of this type should be done to confirm its findings. Any method of treatment for patients with systemic lupus erythematosus which helps replace or reduce drug treatment with all its potential side effects is to be welcomed.

AJEW wishes to thank 7 Seas Health Care for providing Max EPA and olive oil capsules, NE Thames RHA for financial support, and Mova de Wet for further dietary assistance. WJWM wishes to thank the Muir-Hambro Trust Roval College of Physicians for financial support.

1 Homsy J, Morrow W J W, Levy J A. Nutrition and autoimmunity: a review. Clin Exp Immunol 1986; 65: 473-88.

2 Prickett J D, Robinson D R, Steinberg A D. Effects of dietary enrichment with eicosapentaenoic acid upon autoimmune nephritis in female $N Z B \times N Z W=F$, mice. Arthritis Rheum 1983; 26: 133-9.

3 Robinson D R, Prickett J D, Makoul G T, Steinberg A D) Colvin R B. Dietary fish oil reduces progression of estab lished renal disease in $\mathrm{NZB} \times \mathrm{NZW} \mathrm{F}_{1}$ mice and delavs renal disease in BXSB and MRL 1 strains. Arthritis Rheum 1986; 29: 539-46.

4 Kelly V E, Ferretti A, Izui S, Strom T B. A fish oil diet rich in eicosapentaenoic acid reduces cvclooxygenase metabolites and suppresses lupus in MRL-1pr mice. F Immunol 1985: 134: 1914-9.

5 Godfrey D G, Stimson W H, Watson J Sturrock R D. Effects of dietary supplementation on autoimmunity in the MRL lpr mouse: a preliminary investigation. Ann Rheum Dis 1986; 45: 1019-24.

6 Corman L C. The role of diet in animal models of systemic lupus erythematosus: possible implications for human lupus. Semin Arthritis Rheum 1985; 15: 61-9.

7 Sperling R I, Weinblatt M, Robin J-L, et al. Effects of dietary supplementation with marine fish oil on leukocvte lipid mediator generation and function in rheumatoid arthritis. Arthritis Rheum 1987; 30: 988-97.

8 Darlington I. G, Ramsey N W, Mansfield J R. Placebocontrolled blind study of dietary manipulation therapy in controlled blind study of dietary manipulation

9 Belch J J F. Ansell D, Madhok R, O'Dowd A, Sturrock R D. Effects of altering dietary essential fatty acids on require ments for non-steroidal anti-inflammatory drugs in patient with rheumatoid arthritis: a double blind placebo con trolled study. Ann Rheum Dis 1988; 47: 96-104

10 Tan E M, Cohen A S, Fries J F. The 1982 revised criteria for the classification of systemic lupus ervthematosus. Arthrit Rheum 1982; 25: 1271-7.

11 Edwards J C W. Snaith M L. Isenberg I) A. A double blind controlled study of high dose versus low dose methyl prednisolone infusions in sistemic lupus ervithematosus. prednisolone infusions in sistem
Ann Rheum Dis 1987; 46: 733-6.

12 Folch J, Lees M. Sloane Stanley G H. A simple method for the isolation and purification of total lipids from animal tissues. F Biol Chem 1957: 226: 497-509.

13 Morrison W R. Smith L M. Preparation of fatty acid methyl esters and dimethvlacetvls from lipids, with boron fluoride methanol. I Lipid Res 1964: 5: 600-8.

14 Ziff $M$. Diet in the treatment of rheumatoid arthritis. Arthrits Rheum 1983; 26: 457-61.

15 Dong C H, Banks J. The arthritis cookbook. New York: Bantam (Thomas Y Crowell Company), 1973.

16 Dong C H, Banks J. New hope for the arthritic. New York: Ballentine (Thomas Y Crowell Company), 1975.

17 Panush R S, Carter R L, Kat\% P, Kowsari B, Longley S, Finnie S. Diet therapy for rheumatoid arthritis. Arthriti Rheum 1983; 26: 462-71.

18 Darlington L. G. Do diets rich in polyunsaturated fatty acid affect disease activity in rheumatoid arthritis? Ann Rheum Dis 1988; 47: 169-72.

19 Kremer J M, Bigauoette J, Michalek A V. et al. Effects of manipulation of dietary fatty acids on clinical manifestations of rheumatoid arthritis. Lancet 1985; i: $184-7$.

20 Birtwistle S, McEwen L M. Dietary fatty acids and rheumatoid arthritis. Lancet 1985; i: 700 .

21 van der Tempel $H$, Tulleken J E, Limburg P C, Muskier F A J, van Rijswijk M H. Effects of fish oil supplementaF A J, van Rijswijk $M$ H. Effects of fish oil supplementation in

22 Thorner A, Walldius G, Nilsson B, Hadell K, Gullberg R. Beneficial effects of reduced intake of polyunsaturated fatty acids in the diet for one year in patients with systemic lupus erythematosus. Ann Rheum Dis 1990; 49: 134

23 Liang M H, Socher S A, Larson M G, Shore P H. Reliability and validity of 6 systems for the clinical assessment of disease activity in SLE. Arthritis Rheum 1989; 32: 1107-18.

24 Symmons D P M, Coppock J S, Bacon P A, et al. Development and assessment of a computerised index of clinical disease activity in systemic lupus erythematosus. $O \mathcal{F ~ M e d}$ 1988; 69: 927-37.

25 Morrow W J W, Swanson C A, Homsy J, Levy J A. The influence of nutrition on experimental autoimmune disease. An overview with particular emphasis on the effects of dietary fat. In: Cunningham-Rundles S, ed. Nutritional regulation of immune responses. New York: Marcel regulation of immu
Dekker, (in press).

26 Robinson D R, Tateno S, Knoell C, et al. Dietary marine lipids suppress murine autoimmune disease. F Interm Med 1989; 225: 211-4. 